\title{
When 'negativity' becomes obstructive: a novel exploration of the two-factor model of the Self-Compassion Scale and a comparison of self-compassion and self-criticism interventions
}

\author{
Michail Mantzios (DD A,C,D,E,F, Anastasija Koneva ${ }^{A, B, C, D, E, F}$, Helen Egan (DD ${ }^{D, E}$ \\ Birmingham City University, Birmingham, United Kingdom
}

\section{BACKGROUND}

Self-compassion is a tendency to respond to personal feelings of distress in a kind and understanding way, and to become aware that facing difficulties and adversity is part of a common human experience that is shared by all humans. The Self Compassion Scale (SCS) includes negative items measuring self-judgement, isolation and over-identification, which are at the opposite end of the spectrum to self-kindness, common humanity and mindfulness. Some researchers have argued that the link between self-compassion and psychopathology is inflated by the inclusion of these negative items. Moving away from factorial structures and advanced statistics used in recent research, we present a different way of exploring the conceptualisation of self-compassion theory and the way it is measured.

\section{PARTICIPANTS AND PROCEDURE}

Study 1 set out to support the inclusion (or exclusion) of the negative items within the SCS, by investigating the correlation between the negative items and the positive items altered to reflect the exact opposite of the original positive items of the scale (i.e., self-unkindness, disjointed humanity, and mindlessness). Study 2 was an experiment exploring differences between self-compassion and self-criticism 5-minute interventions on state self-compassion, state mindfulness and state anxiety. The interventions were separated to represent the positive or negative elements, rather than a mixture of the interventions.

RESULTS

If the main argumentation against the use of the overall score of the self-compassion scale is the inflation of the negative items, then the results support the inclusion of the negative items within the SCS, as the altered positive items show a similar inflation to the original negative items when observing a significant positive relationship. No differences were found between the two interventions and the overall scores; nevertheless, mindfulness and selfjudgment subscales appeared to significantly change only for the self-compassion group.

\section{CONCLUSIONS}

While the debate around the self-compassion scale continues, the literature emphasizing self-criticism does not translate into inflation as suggested, and does not propose effective practices. Explanations of findings, limitations and suggestions for future research are discussed.

\section{KEY WORDS}

self-compassion; self-compassion scale; self-criticism; interventions

Corresponding Author - Michail Mantzios, Ph.D., Birmingham City University, The Curzon Building Room C307,

B4 7BD Birmingham, United Kingdom, e-mail: michael.mantzios@bcu.ac.uk

Authors' contribution - A: Study design · B: Data collection · C: Statistical analysis · D: Data interpretation ·

E: Manuscript preparation · F: Literature search · G: Funds collection

to Cite this ARTICle - Mantzios, M., Koneva, A., \& Egan, H. (2020). When 'negativity' becomes obstructive: a novel exploration of the two-factor model of the Self-Compassion Scale and a comparison of self-compassion

and self-criticism interventions. Current Issues in Personality Psychology, 8(4), 289-300.

RECEIVED 23.10.2019 • REVIEWED 10.01.2020 • ACCEPTED 06.10.2020 • PUBLISHED 09.11.2020 


\section{BACKGROUND}

Self-compassion is a method of directing compassion towards oneself. According to Neff (2003a, b), selfcompassion is defined as a tendency to be understanding and compassionate towards oneself in the face of personal shortcomings. Self-compassion includes three interrelated components: self-kindness, mindfulness and common humanity (Neff, 2003a). Neff (2003a) further described how self-kindness, mindfulness, and common humanity have polar opposites such as self-judgment, over-identification, and isolation, correspondingly, all coming together to form the tendency to be compassionate towards oneself.

The tool used for measuring self-compassion as defined above is the Self Compassion Scale (SCS; Neff, 2003b). This scale contains six interrelated factors: three negative subscales (self-judgement, over-identification and isolation) and three positive subscales (self-kindness, mindfulness and common humanity). In the original development of the scale, the negative components were conceptualised as merely the opposites of the positive components, as a method of minimising response bias and to relate the items that may be more familiar to Western cultures (Neff, 2016a, b). People in Western cultures are undeniably more exposed to negative aspects that are evident in the SCS (Neff \& Dahm, 2015). It is common, for example, to be critical and judgmental of personal mistakes and failures as a way to develop and excel in personal and professional lives. Exploration of each factor independently or the overall self-compassion score by combining the scores from each of the subscales after reversing the negative items (Neff, 2003b) was common practice, but utilising the scale this way has been questioned.

The positive components of the scale are consistent with the caring aspects someone would expect in any theory of self-compassion (see Mantzios \& Egan, 2017; Egan \& Mantzios, 2018 for review), but the negative components may represent a separate concept, especially when exploring literature that has outlined the association between self-compassion and psychopathology (Muris, Otgaar, \& Petrocchi, 2016). The use of the scale, its psychometric validity, theoretical consistency, and the factor model have been questioned (e.g., Williams, Dalgleish, Karl, \& Kuyken, 2014), which led many researchers to use the scale in different ways. Specifically, researchers suggested that the negative items correlate more with psychopathology, which led them to the conclusion that positive and negative items should be investigated separately, as the negative items inflate the relationships observed (López et al., 2015). Based on the assumption that the negative items represent a different construct, computing all of the items to calculate a total SCS score was considered methodologically flawed (e.g., Williams et al., 2014). The solution proposed was the collapsing of the scale into a two-factor model of the three negative subscales (self-judgement, overidentification and isolation) representing a construct of being "self-critical" and the three positive subscales (self-kindness, mindfulness and common humanity) representing the construct of "self-compassion" (Costa, Marôco, Pinto-Gouveia, Ferreira, \& Castilho, 2015; Muris \& Petrocchi, 2016). Most research has explored the factor structure, which led to the appropriate conclusions, but other research supported the use of the SCS the way it was originally developed and proposed.

Then again, the two-factor model has not been confirmed in other research (e.g., Cleare, Gumley, Cleare, \& O'Conner, 2018). Neff, Whittaker, and Karl (2017) argued for another statistical approach that was more considerate of multiple and overall scores in psychometric scales, and in this case, more appropriate for the SCS. Tóth-Király, Bőthe, and Orosz (2017) further argued that an alternative statistical approach (as suggested by Neff et al., 2017) with exploratory structural equation modelling (ESEM) could provide the highest consistency to theoretically examine the multidimensional nature of the SCS. Adopting such a model, Tóth-Király et al. (2017) examined the factor structure in a large international investigation of 20 samples $(N=11,685)$, where each sample provided support for the use of the original six subscales and the total SCS score, but not the two-factor model. While Neff (2019) attempted to support the original factor structure in more recent literature, the debate continues (Muris \& Otgaar, 2020), and this research aimed to provide an alternative assessment to such debate.

\section{THE PRESENT STUDIES}

The current work encompasses two studies. Study 1 provides an alternative method of examining the question about the factor structure and the overall score of the SCS. Based on the debate around the two-factor model, we took the positive subscale items of the SCS and rephrased them to create self-unkindness, disjointed humanity, and mindlessness, and explored the association of this altered version of the sub-scales to the original positive and negative factors. While previous research has looked at a variety of factorial models of the scale, no research has attempted to alter the positive items to create mirror items that reflect the negative version, and explore the potential similarities to the negative factors proposed in the original scale. Study 1 aimed to examine the use of the three negative subscales, and support the inclusion/exclusion in the overall score for the SCS based on the potential inflation if Neff (2003a, b) had used the exact opposite of the proposed positive items. In essence, we were interested in finding out whether simply negatively worded items representing the polar opposites (as seen in other psychometric scales; e.g., Baer et al., 2008; Lee 
\& Ashton, 2004) would similarly inflate the scores of the scale as observed in past research that utilised the original scale. We hypothesised that a high positive correlation would be observed between original negative items and altered positive items, as well as a high negative correlation between original positive items and reversed positive items. While exploring items of the self-compassion scale presents one challenge, another challenge rests on the literature around self-criticism. Minimising or enduring the presence of self-criticism is where much significance rests in the literature around self-compassion, as self-criticism components indicate significant maladaptive elements across different clinical conditions (e.g., Dunkey \& Grilo, 2007; Rosenfarb, Becker, Khan, \& Mintz, 1998). Enhancing our understanding whether the proposed original negative factors represent polar opposites is one way of inquiry, while another is exploring whether utilising positive over negative elements in compassion-based interventions is best practice.

Study 2 was designed to potentially provide further support for the therapeutic benefits of the selfcompassion intervention, which has been evident in several studies (Albertson, Neff, \& Dill-Shackleford, 2015; Diedrich, Hofmann, Cuijpers, \& Berking, 2016; Johnson \& O'Brien, 2013; Mosewich, Crocker, Kowalski, \& DeLongis, 2013; Neff, 2016a; Odou \& Brinker, 2014). To evidence self-compassion practices as being central to enhancing peoples' perceptions of themselves, we compared the "Self-Compassion Break" to a similarly timed "Self-Criticism Reduction Break" (both developed purposefully for this research to exclude any overlap between positive and negative guidance). Guiding people to be less critical during meditation may not be the best way to purposefully provide guidance, as there is the possibility of enhancing suppression, control, judgment and failure (e.g., Tsal \& Makovski, 2006) without the all-important acceptance and non-judgmental aspects of self-compassion (i.e., self-kindness, mindfulness and common humanity). Another important aspect that bares consideration is that suppressing thoughts and emotions, which often comes with attempts to change thoughts, typically leads to a paradoxical increase, rather than a decrease of thoughts and emotions (Beevers, Wenzlaff, Hayes, \& Scott, 1999; Dalgleish, Yiend, Schweizer, \& Dunn, 2009). Interestingly, research has indicated that suppression leads to more thoughts and more distress, while acceptance of thoughts leads to fewer thoughts, as well as lower levels of depression and anxiety (Marcks \& Woods, 2005). While self-compassion suggests a model of acceptance, a reducing selfcriticism intervention may present a methodological challenge in reducing the negative aspects that have been highlighted to be significant in psychopathology. In other words, guiding people how to think and feel may be better than guiding them how to not think and feel, and the only way forward may be through the positive aspects of self-compassion, especially through interventions. The aim of Study 2 was to explore the two-factor model through interventions, and find support for (or against) the use of self-compassion interventions when compared to the use of a self-criticism reduction intervention. To demonstrate the differences in effectiveness of the interventions, we measured participant outcomes such as state mindfulness, self-compassion and anxiety in an experimental setting. We hypothesised that following the interventions, the selfcompassion intervention would display higher scores in state mindfulness and self-compassion and lower in anxiety over the self-criticism reduction intervention.

\section{STUDY 1}

\section{PARTICIPANTS AND PROCEDURE}

\section{PARTICIPANTS}

A total of 155 undergraduate students from a university in England participated in Study 1. The responses were collected over a period of 6 weeks. The sample consisted of 141 females and 14 males, with a mean age of $20.56(S D=2.86)$. Information about participants also indicated that 134 participants were familiar with the concept of self-compassion and 31 participants practised meditation either currently or in the past. Participants were recruited using voluntary sampling, through a Research Participation Scheme within the university and were rewarded with research credits. Ethical approval for this study was provided by the university's Psychology Department Ethics Committee.

\section{MATERIALS AND MEASURES}

The original Self-Compassion Scale (Neff, 2003b). This is a 26-item scale that measures self-compassion using 3 positive subscales (self-kindness, mindfulness and common humanity) and 3 negative subscales (self-judgement, over identification and isolation). The scale asks participants to rate how they typically act towards themselves in difficult times. For example, a negative item is: "I'm disapproving and judgmental about my own flaws and inadequacies", and a positive item is: "When things are going badly for me, I see the difficulties as part of life that everyone goes through". Participants respond on a 5-item Likert-type scale from 1 (almost never) to 5 (almost always). In the current study the Cronbach's $\alpha$ was .95 .

The Altered Self-Compassion Negative Sub-Scales (Koneva \& Mantzios, 2018; see Appendix). This subscale consisted of 13 additional items, which were randomly inserted into the original Self-Compassion Scale (Neff, 2003b). The additional items were created 
by reversing all positive items from the original scale. For example, "When things are going badly for me, I see the difficulties as part of life that everyone goes through" was altered to "When things are going badly for me, I don't see the difficulties as part of life that everyone goes through". To keep the items as close to the original as possible, we added to each altered question only 1-2 words to phrase it negatively. In the current study the Cronbach's $\alpha$ was .84. The original scale with the added altered sub-scale was a 39-item scale, composed of 9 subscales: self-kindness, common humanity, mindfulness, self-judgement, isolation, overidentification, reversed self-kindness, reversed common humanity and reversed mindfulness. Responses were given on a 5-point Likert-type scale from 1 (almost never) to 5 (almost always). In the current study the Cronbach's $\alpha$ was .95 for the combined scale.

\section{DESIGN AND PROCEDURE}

Study 1 was set up online and recruitment occurred though the Research Participation Scheme of the university. Participants were provided with a link, where they were presented with a participant information sheet and a consent form. They were first required to fill in a demographics questionnaire and then to complete the self-compassion items. After the completion, participants were provided with a debriefing sheet. Correlational design was used in this study and negative items from the original scale were compared to positive and reversed positive items.

\section{RESULTS AND DISCUSSION}

Preliminary analysis showed that all assumptions were met to conduct a bivariate correlation. The relationship between original positive $(\mathrm{O}+)$, original negative $(\mathrm{O}-)$ and altered $(\mathrm{A})$ subscales was investigated using Pearson correlation coefficient. There was a strong negative correlation between $\mathrm{O}+$ and $\mathrm{O}-$ subscales $(r=-.69, n=155, p<.001)$, a strong negative correlation between $\mathrm{O}+$ and A subscales $(r=-.68, n=155$, $p<.001)$, and a strong positive correlation between O- and A subscales $(r=.72, n=155, p<.001)$.

The SCS appears to be an appropriate measure as suggested by Neff (2003b), as the relationships appeared to be as predicted. Specifically, the inclusion of the negative components for the reduction of response bias appears appropriate, as there is a significant positive relationship between $\mathrm{A}$ items and $\mathrm{O}-$ items, and the $\mathrm{O}+$ items significantly relate negatively to both O- and A, a relationship that appears marginally the same. For now, it appears as if the self-compassion scale represents a valid construct, although replication of the present findings is warranted. The question now is whether the inflation observed in past re- search (López, Sanderman, \& Schroevers, 2018; Muris \& Petrocchi, 2016) is acceptable, which is something that will be addressed in detail in the general discussion. Then again, we considered a completely different methodology to explore the debate around the non-inclusion of the self-critical items, where we explored the differing outcomes that could be produced by self-criticism reduction interventions, rather than the conventional self-compassion intervention observed across the literature.

\section{STUDY 2}

To our knowledge, no other study has reported the effectiveness of an intervention that focuses on selfcompassion, intentionally excluding any mention of the negative components that are originally part of the theory of self-compassion, to compare to a selfcriticism reduction intervention. Similarly, no other research has attempted to explore the potential of an intervention that focuses on reducing the negative components, without the inclusion of the positive components of the theory. We explored the differing outcomes that could be produced by a self-criticism reduction intervention, rather than a conventional self-compassion intervention on outcome variables relating to self-compassion, mindfulness and anxiety. We assumed that non-significant differences in outcomes (i.e., self-compassion, mindfulness and anxiety) between the two interventions would partially suggest that the effectiveness rests on both negative and positive items. On the other hand, it would question the directionality of interventions that is centred more on the positive elements (and items) of self-compassion. Then again, when exploring the effectiveness of self-compassion, and the scale representing self-compassion, it would be expected that the selfcompassion intervention would perform better on the self-compassion scale. Also, considering the inflation of the negative items observed in relation to psychopathology, if anxiety is significantly reduced in the self-criticism reduction intervention when compared to the self-compassion intervention, it would verify the influence of negative items and position results towards the exclusion of the self-criticism items in future research. Again, considering the directionality of self-compassion interventions, it may potentially suggest diverging directions, dependent on individual wellbeing and mental health dictating the appropriate intervention. Last, but not least, if we were to assume that the positive and negative items are polar opposites, we would expect that both self-compassion and self-criticism reduction interventions would influence all self-compassion subscales similarly, rather than self-compassion influencing positive subscales, and the self-criticism reduction intervention influencing negative subscales. Overall, considering that the 
significance rests on the potential of interventions in the field, it is important to explore the significance of self-criticism components in isolation from positive components, and potentially explore the development of interventions and appropriate assessment.

\section{PARTICIPANTS AND PROCEDURE}

\section{PARTICIPANTS}

A total of 80 psychology undergraduate students from a university in England participated in Study 2. They were recruited using volunteer sampling, through a Research Participation Scheme within the university and rewarded with credits. The sample consisted of 76 females ( $n=37$ in self-criticism condition) and 4 males ( $n=3$ in self-criticism condition) with mean age of $21.66(S D=5.07)$. In addition, 65 participants were familiar with the concept of selfcompassion, and 17 participants practised meditation currently or in the past. Half of the participants were assigned to the self-compassion condition and half to the self-criticism condition. Every second participant was allocated to the self-criticism condition, and each condition consisted of 40 participants. Gender differences were explored, and data were reanalysed by excluding males, and similar comparisons were performed with (un)familiarity of self-compassion and experiences in meditation, all of which indicated the same pattern or non-significant differences. Ethical approval for this study was provided by the university's Psychology Department Ethics Committee.

\section{MATERIALS AND MEASURES}

Demographic information such as age, gender and previous knowledge and practising of mindfulnessand compassion-based interventions was collected. In regards to the scales that were used for the present research, we utilised state scales that are capable of measuring changes across smaller timeframes (see e.g., Marteau \& Bekker, 1992).

State Self-Compassion Scale (Breines \& Chen, 2013). The scale measures state self-compassion. It is a 16-item scale with six subscales, including self-kindness, mindfulness, common humanity, self-judgement, over-identification and isolation. Significantly for the present research, this state scale follows the same theoretical underpinning of Neff's trait self-compassion scale, and enables a parallel exploration of the construct. The overall score of the 16 items was explored as an overall score, and the 6 factors were explored with items such as "I'm trying to be kind and reassuring to myself". Responses are given on a 6-point Likert-type scale from 1 (strongly disagree) to 6 (strongly agree). This scale was used to measure state self-compassion of participants prior to (Cronbach's $\alpha=.88$ ) and after intervention (Cronbach's $\alpha=.83$ ).

State Mindfulness Scale (SMS; Tanay \& Bernstein, 2013). The scale measures being aware of experiences and emotions in the present moment. It is a 21-item scale with 2 subscales, Mind and Body. The scale asked participants to rate how well the statements, such as "I was aware of different emotions that arose in me", describe their experiences in the past $15 \mathrm{~min}$ utes. Responses were given on a 5-point Likert-type scale from 1 (not at all) to 5 (very well). This scale was used to measure participants' awareness of present experiences prior to (Cronbach's $\alpha=.95$ ) and after intervention (Cronbach's $\alpha=.95$ ).

State Anxiety Inventory - Short Form (STAI-SF; Marteau \& Bekker, 1992). This scale measures the state anxiety of a participant. It is a 6-item scale that asks participants to rate each statement related to how they feel at the moment. An example of a statement is: "I feel calm". The responses are given on a 4-point Likerttype scale from 1 (not at all) to 4 (very much). The scale was administered both prior to (Cronbach's $\alpha=.87$ ) and after intervention (Cronbach's $\alpha=.88$ ) to measure the changes in anxiety of participants.

\section{INTERVENTIONS}

The interventions used in this study were recorded by a qualified mental health practitioner and counsellor, with experience in practising and teaching meditation. The intervention was either the 5:11 minute SelfCompassion Break (Mantzios, 2017b), which was designed to increase participants self-compassion, or the 5:21 minute Eliminating Self-Criticism Break (Mantzios, 2017a), which was designed to decrease participants' self-criticism. Since there was no "Eliminating Self-Criticism Break" intervention, and the research required that the duration be similar across both conditions, as well as providing the same instructor voice, both interventions were developed for the present research. For the Eliminating Self-Criticism Break, participants were asked to bring into awareness any suffering that they had recently experienced and use it as an example to reflect upon for the practice. Once participants were thinking of the experience, different phrases covered the negative components of self-criticism such as "get yourself to a point where you are not over-identifying or ruminating over the suffering of the situation", "overcoming the isolation of that suffering is key to eliminating self-criticism" and "trying to be less judgmental with yourself as you would be with a friend may enable you to be less critical with your experience". Similarly, the Self-Compassion Break similarly invited participants to bring into awareness a recent moment of suffering and used phrases such as "become aware of this experience of suffering in this moment by approaching it instead of 
avoiding it", "acknowledge that all human beings are suffering, and it is common to experience suffering as all people do" and "express kindness and understanding to yourself as you would with a friend who is suffering”. Participants were also provided with information on positive components of self-compassion in the self-compassion condition prior to intervention, and information on negative components of self-compassion in the self-criticism condition prior to the intervention. Information generically described the three corresponding components, either positive or negative, depending on the intervention. The information was provided to increase participants' understanding of positive and negative sides of self-compassion for corresponding conditions, and to increase the effectiveness of the interventions.

\section{DESIGN AND PROCEDURE}

Participants were invited into the laboratory and were assigned to one of two conditions: self-compassion or self-criticism. After reading the participant information sheet, and signing the consent form, they were instructed to complete the demographics questionnaire and the first set of questionnaires, including the SCS, SMS and STAI. When participants finished completing the state scales, they read the provided information and then listened to the audio recording via headphones. After the audio finished, participants were asked to complete the second set of questionnaires, which consisted of the state scales they completed at baseline. This completed the participation and participants were thanked and debriefed prior to leaving the laboratory. Three 2 (Condition: compassion, criticism) x 2 (Time: pre-, post-intervention) ANOVAs were conducted on state self-compassion, state mindfulness and state anxiety. Similar analyses were conducted on each subscale of the self-compassion scale, to enable a more in-depth exploration of similarities and differences in outcomes.

\section{RESULTS AND DISCUSSION}

Preliminary analysis showed no violation of assumptions (i.e., Levene's test of equality of error variances $>.05$ and Box's test of equality of covariance matrices $>.001)$. The analyses revealed that the effects of the intervention on state mindfulness, on state selfcompassion, and on state anxiety were non-significant. There were no significant difference between the intervention types and between genders at baseline measurements. However, there were similar outcomes in increasing self-compassion, state mindfulness and in decreasing state anxiety (see Tables 1 and 2).

Table 1

Summary of mixed ANOVA results for comparison between self-compassion and self-criticism conditions at preand post-test

\begin{tabular}{lccccccc}
\hline State SCS Sub-scales & $d f$ & \multicolumn{3}{c}{ Within-subject effects } & \multicolumn{2}{c}{$\begin{array}{c}\text { Between-subject } \\
\text { effects }\end{array}$} \\
\cline { 3 - 8 } & & \multicolumn{2}{c}{ Time } & \multicolumn{2}{c}{ Time * Group } & Group \\
\cline { 3 - 8 } & & $F$ & $p$ & $F$ & $p$ & $F$ & $p$ \\
\hline State Mindfulness & 1,78 & 59.21 & $<.001$ & 0.34 & .565 & 0.12 & .726 \\
State Self-Compassion & 1,78 & 48.63 & $<.001$ & 2.03 & .158 & 2.49 & .119 \\
State Anxiety & 1,78 & 8.21 & .005 & 0.15 & .697 & 0.15 & .697 \\
\hline
\end{tabular}

Table 2

Mean difference of pre-and post-intervention scores by condition

\begin{tabular}{|c|c|c|c|c|c|c|}
\hline Measure & Condition & $M(S D)$ & $M(S D)$ & $\Delta M$ & $t$ & $p$ \\
\hline \multirow[t]{2}{*}{ State Mindfulness } & Self-Criticism & $64.50(18.54)$ & $77.73(19.96)$ & 13.23 & -4.53 & $<.001$ \\
\hline & Self-Compassion & $64.63(17.36)$ & $80.00(14.13)$ & 15.38 & -6.59 & $<.001$ \\
\hline \multirow{2}{*}{$\begin{array}{l}\text { State } \\
\text { Self-Compassion }\end{array}$} & Self-Criticism & $77.55(11.64)$ & $82.85(11.15)$ & 5.30 & -4.66 & $<.001$ \\
\hline & Self-Compassion & $71.85(16.16)$ & $79.88(11.87)$ & 8.03 & -5.23 & $<.001$ \\
\hline \multirow[t]{2}{*}{ State Anxiety } & Self-Criticism & $11.25(4.34)$ & $10.30(4.08)$ & -0.95 & 1.89 & .067 \\
\hline & Self-Compassion & $12.63(3.68)$ & $11.38(3.84)$ & -1.25 & 2.16 & .037 \\
\hline
\end{tabular}


Table 3

Summary of mixed ANOVA results for comparison between self-compassion and self-criticism conditions at preand post-test

\begin{tabular}{lccccccc}
\hline State SCS Sub-scales & $d f$ & \multicolumn{3}{c}{ Within-subject effects } & \multicolumn{2}{c}{$\begin{array}{c}\text { Between-subject } \\
\text { effects }\end{array}$} \\
\cline { 3 - 9 } & & \multicolumn{2}{c}{ Time } & \multicolumn{2}{c}{ Time * Group $^{*}$} & Group \\
\cline { 3 - 8 } & & $F$ & $p$ & $F$ & $p$ & $F$ & $p$ \\
\hline Self-Kindness & 1,78 & 71.28 & $<.001$ & 0.25 & .617 & 2.48 & .120 \\
Self-Judgment & 1,78 & 8.79 & .004 & 2.25 & .138 & 0.97 & .328 \\
Common Humanity & 1,78 & 14.53 & $<.001$ & 0.61 & .438 & 5.19 & .025 \\
Isolation & 1,78 & 17.55 & $<.001$ & 0.36 & .551 & 0.57 & .452 \\
Mindfulness & 1,78 & 2.36 & .129 & 7.29 & .009 & 2.18 & .144 \\
Over-Identification & 1,78 & 0.99 & .322 & 1.39 & .243 & 1.44 & .233 \\
\hline
\end{tabular}

Therefore, the self-compassion and self-criticism interventions do not differ in the way they influence state self-compassion, state mindfulness and state anxiety, although state anxiety appeared to reach significance in the Self-Compassion Condition, while the Self-Criticism Condition marginally failed. Having a primary interest in self-compassion, the subscales were further investigated, in an attempt of exploring the data fully. Table 3 shows a summary of mixed analyses of variance conducted on each subscale of the self-compassion scale.

Significant findings were followed up with repeated measures $t$-tests. For the Compassion Condition, isolation was significantly reduced from pre- to post-intervention measurements, $t(78)=2.54, \Delta M=1.05, p=.013$. Similarly, for the Criticism Condition, isolation was significantly reduced from pre- to post-intervention measurements, $t(78)=3.39, \Delta M=1.40, p=.001$. Findings were similar for Common Humanity and SelfKindness. Common Humanity for the Compassion Condition displayed a significant increase $t(78)=3.25$, $\Delta M=-1.40, p=.002$, and the same was true for the Criticism Condition $t(78)=2.14, \Delta M=-0.92, p=.035$. Self-Kindness for the Compassion Condition displayed a significant increase $t(78)=5.62, \Delta M=-2.37, p<.001$, and a similar increase was observed for the Criticism Condition $t(78)=6.32, \Delta M=-2.67, p<.001$. Up to this point, there were no differences between the two conditions. For Mindfulness and Self-Judgment, however, the findings indicated semantic differences. For mindfulness, the Compassion Condition displayed a significant increase $t(78)=2.99, \Delta M=-1.00, p=.004$, while the Criticism Condition displayed a non-significant decrease, $t(78)=0.82, \Delta M=0.27, p=.413$. Similarly, self-judgment significantly decreased for the Compassion Condition, $t(78)=3.16, \Delta M=1.60, p=.002$, while the Criticism Condition displayed a non-significant decrease, $t(78)=0.53, \Delta M=0.53, p=.304$.

\section{DISCUSSION}

The current study set out (a) to investigate the accuracy of the SCS for measuring self-compassion and to provide further support for the inclusion/exclusion of the negative items within the scale, and (b) to further explore the rationale of the SCS by showing the difference in state variables between a self-compassion vs. reducing self-criticism intervention. The first hypotheses were verified, and the study found a strong positive correlation between the original negative subscale of the SCS and the altered subscale. In other words, whether we are talking about the much debated negative items or the positive items rephrased (to represent negative items - i.e., the exact polar opposites), there seems to be an overly high association, which translates into being more similar than different. In addition, a strong negative correlation between the original positive subscale and the altered subscale was observed. The second hypothesis was also confirmed, but not to the extent that was expected. The main analyses showed no significant difference between the two interventions across all measures used, apart from a marginal difference in state anxiety. Follow-up analyses into the subscales revealed that there were significant differences in self-judgment and mindfulness. This means that the significance was limited when exploring the differences in the effectiveness of state self-compassion, state mindfulness and state anxiety.

The findings of Study 1 supported the first hypothesis. The current study showed a strong correlation between the negative items and reversed-positive items, suggesting that the negative components are simply the opposites of the positive components. Study 1 contributed to the understanding of factor models that can be used with the SCS. While studies have shown the suitability and problems of the 
six-factor model (Castilho, Pinto-Gouveia, \& Duarte, 2015; Williams et al., 2014), the overall self-compassion score (Neff et al., 2018), and the two-factor model (Costa et al., 2015; Toth-Kiraly et al., 2017), the current study indicates that the use of negative items within the SCS is acceptable. Study 1 supports the understanding that while negative and positive components might be opposites and relate differently to other constructs, they are interrelated parts of an overall concept of self-compassion. In essence, the contribution or inflation of the negative items is not much more than that of positive items rephrased.

Previous research indicated that positive and negative items within the scale represent separate concepts, because negative items have a stronger association with psychopathology (e.g. López et al., 2018; Muris \& Petrocchi, 2016). However, this could be explained by factors other than negative components being more predictive of psychopathology. For example, researchers have claimed that people from Western civilisation relate more easily to the negative components of self-compassion than the positive ones (Neff \& Dahm, 2015). The culture of the West teaches people to associate self-compassion with passivity or weakness and to confuse the concept with self-indulgence or self-pity, rather than a healthier perspective of oneself that is able to battle psychopathology (Egan \& Mantzios, 2018; Mantzios \& Egan, 2017; Neff \& Dahm, 2015). In addition, people struggling with mental health, such as those who show symptoms of depression, find the concept of self-compassion difficult to practise and components of self-compassion difficult to experience (Pauley \& McPherson, 2010). This could contribute to the stronger link between negative items and psychopathology. The present research uniquely contributed to the present literature by indicating that the exact opposite to the positive items, which is seen in most psychometric tools (e.g., Baer et al., 2008; Lee \& Ashton, 2004), is equivalent to the original negative items. This research suggests that people more generally perceive the negative items as the exact opposite to the positive ones, by exploring the relationship between positive items that were altered and the controversial original negative items. While findings in Study 1 appear to add some additional clarity to the debate, the findings of the second study are clear, but raise further questions.

Study 2 did not support the second hypothesis of the current study as expected. The analysis showed no significant difference between the interventions. Both interventions were equally effective at increasing the overall state self-compassion and state mindfulness and decreasing state anxiety scores, although state anxiety showed a marginal significance of a higher reduction for the self-compassion (over the self-criticism) intervention. Self-compassion has been conceptualised as more than simply a lack of self-criticism (Warren, Smeets, \& Neff, 2016), and the overall scores provided a general overview that was explored further by investigating the subscales. Isolation, Common Humanity and Self-Kindness appeared to provide a similar pattern, whereby both groups non-significantly differed in increasing scores in Common Humanity and Self-Kindness, while both reduced Isolation, explaining the overall self-compassion score being non-significantly different. Nevertheless, Mindfulness and Self-Judgment appeared to be substantially different when exploring pre- and post-measurements. Mindfulness significantly increased in the self-compassion intervention, while decreasing in the self-criticism, non-significantly. Considering mindfulness to be the primary construct of initiating compassion for self and others, as it assists identifying when there is suffering, and the need to enable the appropriate compassionate response, there is much importance in this finding that needs further consideration. With self-judgment, both interventions decreased self-judgment, but the selfcompassionate intervention decreased self-judgment significantly, while the self-criticism intervention did not reach significance. Importantly, self-compassion and the inclusivity of mindfulness create an interrelation that is significant in cognitive enhancement. The ability to improve cognitive appraisal (Chishima, Mizuno, Sugawara, \& Miyagawa, 2018) and reappraisal (Diedrich et al., 2016), as well as cognitive flexibility (Shahabi, Shahabi, \& Forozendeh, 2020), and cognitive outcomes for populations in distress (Allen, Robertson, \& Patin, 2017) are significant considering the widespread use of self-compassion interventions. Again, these findings bare more significance when considering that one of the interventions aimed at reducing self-criticism, and performed worse than the self-compassion intervention. The findings of the current study are not straightforward, but do support the notion that telling people to be less critical of themselves is not the best solution. If this was the case, there would be a far lower demand for mental health research and professionals, as advising and guiding would ensure straightforward avenues to successful intervention in health and wellbeing. The findings relate to previously explored literature, where predictions were based on the assumption of suppression and the paradoxical effect (Beevers et al., 1999; Marcks \& Woods, 2005; Dalgleish et al., 2009) of "over-identifying", but the reality is that there should have been more significant findings. Conversely, the findings could be explained through a recent systematic review and meta-analysis that highlighted selfcompassion interventions improving self-compassion and psychopathology, but not over and beyond other interventions (Wilson, Mackintosh, Power, \& Chan, 2019), which is consistent with the present findings.

The findings could further be explained when considering the use of the short interventions in 
the current study, and future research could look into extending the research by investigating longer durations of meditation, although from an ethical viewpoint the use of decreasing self-criticism for prolonged periods and interventions may be problematic, and was therefore explored in this short format. Similarly, including a self-compassion break as intended by Neff $(2003 a, b)$ may signify how together they pose a superior model of intervention, rather than simply separating them based on the two-factor model, overall creating three conditions that can lead to further clarity in clinical and practical settings.

Importantly, other limitations of the current studies should be acknowledged before drawing any further conclusions. One of the limitations relates to the recruitment of participants. Both studies relied on a Research Participation Scheme within the university to recruit participants, which is based on rewarding participants with credits. There have been indications that reward for participation in psychological studies may negatively influence participants and motivation to engage with the task at hand (Jovanovic \& Matejevic, 2014; Sharp, Pelletier, \& Levesque, 2006). Furthermore, the design of Study 2 required priming participants with the relevant information. Participants in the self-compassion condition were asked to familiarise themselves with the descriptions of the positive components of self-compassion, while those in the self-criticism condition were asked to read information on the negative components, to increase participants' understanding of the relevant message prior to the administration of the intervention. However, all of the participants were psychology undergraduates, and over $80 \%$ of the participants reported being familiar with the concept of self-compassion. This means that their understanding of the concept was more similar across conditions than the design of the study intended. Future research should use a pre-intervention test to assess prior knowledge as a method of excluding participants, to ensure that the results are truly reflective of the interventions used. Lastly, a larger sample size should be aimed for in future research to increase the trust in the data.

In conclusion, the current studies set out to further support the inclusion/exclusion of negative items within the SCS. Limitations notwithstanding, this work, alongside existing research, strengthens the rationale for use of the SCS in its current form in Study 1, but Study 2 may need further consideration. Without any follow-up investigations, the conclusion that can be drawn from Study 2 may only be that the positive item intervention brings about positive changes to both positive and negative factors, but not over and beyond what would be expected. In essence, the second study is not as supportive of the psychometric properties when considering an intervention that was developed strictly based on the positive items. There is a need to highlight the essence of what and why we do what we do as researchers and practitioners. The importance of self-compassion was never the scale itself, but the interventions that came with it, and the ability to help people in need quite similar to mindfulness scales and the imminent benefits of mindfulness practices.

\section{References}

Albertson, E. R., Neff, K. D., \& Dill-Shackleford, K. E. (2015). Self-compassion and body dissatisfaction in women: a randomized controlled trial of a brief meditation intervention. Mindfulness, 6, 444-454. https://doi.org/10.1007/s12671-014-0277-3

Allen, A. B., Robertson, E., \& Patin, G. A. (2017). Improving emotional and cognitive outcomes for domestic violence survivors: The impact of shelter stay and self-compassion support groups. Journal of Interpersonal Violence. https://doi.org/10. 1177/0886260517734858

Baer, R. A., Smith, G. T., Lykins, E., Button, D., Krietemeyer, J., Sauer, S., Walsh, E., Duggan, D., \& Williams, J. M. G. (2008). Construct validity of the Five Facet Mindfulness Questionnaire in meditating and nonmeditating samples. Assessment, 15, 329342. https://doi.org/10.1177/1073191107313003

Beevers, C. G., Wenzlaff, R. M., Hayes, A. M., \& Scott, W. D. (1999). Depression and the ironic effects of thought suppression: Therapeutic strategies for improving mental control. Clinical Psychology: Science and Practice, 6, 133-148. https:// doi.org/10.1093/clipsy.6.2.133

Breines, J. G., \& Chen, S. (2013). Activating the inner caregiver: The role of support-giving schemas in increasing state self-compassion. Journal of Experimental Social Psychology, 49, 58-64. https:// doi.org/10.1016/j.jesp.2012.07.015

Castilho, P., Pinto-Gouveia, J., \& Duarte, J. (2015). Evaluating the multifactor structure of the long and short versions of the self-compassion scale in a clinical sample. Journal of Clinical Psychology, 71, 856-870. https://doi.org/10.1002/jclp.22187

Chishima, Y., Mizuno, M., Sugawara, D., \& Miyagawa, Y. (2018). The influence of self-compassion on cognitive appraisals and coping with stressful events. Mindfulness, 9, 1907-1915. https://doi. org/10.1007/s12671-018-0933-0

Cleare, S., Gumley, A., Cleare, C. J., \& O'Connor, R. C. (2018). An investigation of the factor structure of the Self-Compassion Scale. Mindfulness, 9, 618628. https://doi.org/10.1007/s12671-017-0803-1

Costa, J., Marôco, J., Pinto-Gouveia, J., Ferreira, C., \& Castilho, P. (2015). Validation of the psychometric properties of the Self-Compassion Scale. Testing the factorial validity and factorial invariance of the measure among borderline personality disorder, anxiety disorder, eating disorder and general 
populations. Clinical Psychology \& Psychotherapy, 23, 460-468. https://doi.org/10.1002/cpp.1974

Dalgleish, T., Yiend, J., Schweizer, S., \& Dunn, B. D. (2009). Ironic effects of emotion suppression when recounting distressing memories. Emotion, 9, 744749. https://doi.org/10.1037/a0017290

Diedrich, A., Hofmann, S. G., Cuijpers, P., \& Berking, M. (2016). Self-compassion enhances the efficacy of explicit cognitive reappraisal as an emotion regulation strategy in individuals with major depressive disorder. Behaviour Research and Therapy, 82, 1-10. https://doi.org/10.1016/j.brat.2016.04.003

Dunkley, D. M., \& Grilo, C. M. (2007). Self-criticism, low self-esteem, depressive symptoms, and overevaluation of shape and weight in binge eating disorder patients. Behaviour Research and Therapy, 45, 139-149. https://doi.org/10.1016/j.brat.2006.01.017

Egan, H. H., \& Mantzios, M. (2018). A qualitative exploration of self-kindness and 'treating oneself' 'in contexts of eating, weight regulation and other health behaviours: Implications for mindfulnessbased eating programmes. Frontiers in Psychology, 9, 880. https://doi.org/10.3389/fpsyg.2018.00880

Johnson, E. A., \& O'Brien, K. A. (2013). Self-compassion soothes the savage ego-threat system: Effects on negative affect, shame, rumination, and depressive symptoms. Journal of Social and Clinical Psychology, 32, 939-963. https://doi.org/10.1521/jscp. 2013.32.9.939

Jovanovic, D., \& Matejevic, M. (2014). Relationship between rewards and intrinsic motivation for learning - researches review. Procedia - Social and Behavioral Sciences, 149, 456-460. https://doi.org/ 10.1016/j.sbspro.2014.08.287

Koneva, A., \& Mantzios, M. (2018). The Altered SelfCompassion Negative Sub-Scale. Available at the end of the manuscript.

Lee, K., \& Ashton, M. C. (2004). Psychometric properties of the HEXACO personality inventory. Multivariate Behavioral Research, 39, 329-358. https://doi. org/10.1207/s15327906mbr3902_8

López, A., Sanderman, R., \& Schroevers, M. J. (2018). A close examination of the relationship between self-compassion and depressive symptoms. Mindfulness, 9, 1470-1478. https://doi.org/10.1007/s12 671-018-0891-6

López, A., Sanderman, R., Smink, A., Zhang, Y., Van Sonderen, E., Ranchor, A., \& Schroevers, M. J. (2015). A reconsideration of the Self-Compassion Scale's total score: Self-compassion versus self-criticism. PLoS One, 10, e0132940. https://doi.org/10.1371/ journal.pone.0132940

Mantzios, M. (2017a). Eliminating Self-Criticism Break [audio file]. Available upon request from the author.

Mantzios, M. (2017b). Self-Compassion Break [audio file]. Available upon request from the author.

Mantzios, M., \& Egan, H. H. (2017). On the role of selfcompassion and self-kindness in weight regula- tion and health behavior change. Frontiers in Psychology, 8, 229. https://doi.org/10.3389/fpsyg.2017. 00229

Marcks, B. A., \& Woods, D. W. (2005). A comparison of thought suppression to an acceptance-based technique in the management of personal intrusive thoughts: a controlled evaluation. Behaviour Research and Therapy, 43, 433-445. https://doi. org/10.1016/j.brat.2004.03.005

Marteau, T. M., \& Bekker, H. (1992). The development of a six-item short-form of the state scale of the Spielberger State-Trait Anxiety Inventory (STAI). British Journal of Clinical Psychology, 31, 301-306. https://doi.org/10.1111/j.2044-8260.1992.tb00997.x

Mosewich, A. D., Crocker, P. R., Kowalski, K. C., \& DeLongis, A. (2013). Applying self-compassion in sport: an intervention with women athletes. Journal of Sport and Exercise Psychology, 35, 514-524. https://doi.org/10.1123/jsep.35.5.514

Muris, P., \& Petrocchi, N. (2016). Protection or vulnerability? A meta-analysis of the relations between the positive and negative components of self-compassion and psychopathology. Clinical Psychology \& Psychotherapy, 24, 373-383. https:// doi.org/10.1002/cpp.2005

Muris, P., \& Otgaar, H. (2020). The process of science: a critical evaluation of more than 15 years of research on self-compassion with the Self-Compassion Scale. Mindfulness, 11, 1469-1482. https://doi. org/10.1007/s12671-020-01363-0

Muris, P., Otgaar, H., \& Petrocchi, N. (2016). Protection as the mirror image of psychopathology: Further critical notes on the self-compassion scale. Mindfulness, 7, 787-790. https://doi.org/10.1007/ s12671-016-0509-9

Neff, K. D. (2003a). Self-compassion: an alternative conceptualization of a healthy attitude toward oneself. Self and Identity, 2, 85-101. https://doi. org/10.1080/15298860390129863

Neff, K. D. (2003b). The development and validation of a scale to measure self-compassion. Self and Identity, 2, 223-250. https://doi.org/10.1080/ 15298860390209035

Neff, K. D. (2016a). Does self-compassion entail reduced self-judgment, isolation, and over-identification? A response to Muris, Otgaar, and Petrocchi (2016). Mindfulness, 7, 791-797. https://doi.org/ 10.1007/s12671-016-0531-y

Neff, K. D. (2016b). The self-compassion scale is a valid and theoretically coherent measure of self-compassion. Mindfulness, 7, 264-274. https://doi.org/ 10.1007/s12671-015-0479-3

Neff, K. D. (2019). Setting the record straight about the Self-Compassion Scale. Mindfulness, 10, 200202. https://doi.org/10.1007/s12671-018-1061-6

Neff, K. D., \& Dahm, K. A. (2015). Self-compassion: What it is, what it does, and how it relates to mindfulness. In B. D. Ostafin, M. D. Robinson, 
\& B. P. Meier (Eds.), Handbook of mindfulness and self-regulation (pp. 121-137). New York: Springer.

Neff, K. D., Long, P., Knox, M. C., Davidson, O., Kuchar, A., Costigan, A., Williamson, Z., Rohleder, N., Tóth-Király, I., \& Breines, J. G. (2018). The forest and the trees: Examining the association of self-compassion and its positive and negative components with psychological functioning. Self and Identity, 17, 627-645. https://doi.org/10.1080/ 15298868.2018.1436587

Neff, K. D., Whittaker, T. A., \& Karl, A. (2017). Examining the factor structure of the Self-Compassion Scale in four distinct populations: Is the use of a total scale score justified? Journal of Personality Assessment, 90, 596-607. https://doi.org/10.1080/0 0223891.2016.1269334

Odou, N., \& Brinker, J. (2014). Exploring the relationship between rumination, self-compassion, and mood. Self and Identity, 13, 449-459. https://doi.org/ 10.1080/15298868.2013.840332

Pauley, G., \& McPherson, S. (2010). The experience and meaning of compassion and self-compassion for individuals with depression or anxiety. Psychology and Psychotherapy: Theory, Research and Practice, 83, 129-143. https://doi.org/10.1348/147608309X471000

Rosenfarb, I. S., Becker, J., Khan, A., \& Mintz, J. (1998). Dependency and self-criticism in bipolar and unipolar depressed women. British Journal of Clinical Psychology, 37, 409-414. https://doi. org/10.1111/j.2044-8260.1998.tb01398.x

Shahabi, B., Shahabi, R., \& Foroozandeh, E. (2020). Analysis of the self-compassion and cognitive flexibility with marital compatibility in parents of children with autism spectrum disorder. International Journal of Developmental Disabilities, 66, 282-288. https://doi.org/10.1080/20473869.2019.1573000

Sharp, E. C., Pelletier, L. G., \& Lévesque, C. (2006). The double-edged sword of rewards for participation in psychology experiments. Canadian Journal of Behavioural Science, 38, 269-277. https://doi. org/10.1037/cjbs2006014

Tanay, G., \& Bernstein, A. (2013). State Mindfulness Scale (SMS): Development and initial validation. Psychological Assessment, 25, 1286-1299. https:// doi.org/10.1037/a0034044

Tóth-Király, I., Bőthe, B., \& Orosz, G. (2017). Exploratory structural equation modelling analysis of the Self-Compassion Scale. Mindfulness, 8, 881892. https://doi.org/10.1007/s12671-016-0662-1

Tsal, Y., \& Makovski, T. (2006). The attentional white bear phenomenon: The mandatory allocation of attention to expected distractor locations. Journal of Experimental Psychology: Human Perception and Performance, 32, 351-363. https://doi. org/10.1037/0096-1523.32.2.351

Warren, R., Smeets, E., \& Neff, K. (2016). Self-criticism and self-compassion: Risk and resilience: Being compassionate to oneself is associated with emotional resilience and psychological well-being. Current Psychiatry, 15, 18-33.

Williams, M. J., Dalgleish, T., Karl, A., \& Kuyken, W. (2014). Examining the factor structures of the Five Facet Mindfulness Questionnaire and the SelfCompassion Scale. Psychological Assessment, 26, 407-418. https://doi.org/10.1037/a0035566

Wilson, A. C., Mackintosh, K., Power, K., \& Chan, S. W. (2019). Effectiveness of self-compassion related therapies: a systematic review and meta-analysis. Mindfulness, 10, 979-995. https://doi.org/10.1007/ s12671-018-1037-6 


\section{THE ALTERED SELF-COMPASSION NEGATIVE SUB-SCALES}

1. When things are going badly for me, I don't see the difficulties as part of life that everyone goes through.

2. I don't try to be loving towards myself when I'm feeling emotional pain.

3. When I'm down and out, I don't remind myself that there are lots of other people in the world feeling like I am.

4. When something upsets me I don't try to keep my emotions in balance.

5. When I feel inadequate in some way, I don't try to remind myself that feelings of inadequacy are shared by most people.

6. When I'm going through a very hard time, I don't give myself the caring and tenderness I need.

7. When something painful happens I don't try to take a balanced view of the situation.

8. I don't try to see my failings as part of the human condition.

9. When I fail at something important to me I don't try to keep things in perspective.

10. I'm not kind to myself when I'm experiencing suffering.

11. When I'm feeling down I don't try to approach my feelings with curiosity and openness.

12. I'm not tolerant of my own flaws and inadequacies.

13. I don't try to be understanding and patient towards those aspects of my personality I don't like. 